\title{
A perturbation result for the Riesz transform
}

\author{
BAPTISTE DEVYVER
}

\begin{abstract}
We show a perturbation result for the Riesz transform: if $M_{0}$ and $M_{1}$ are complete Riemannian manifolds which are isometric outside a compact set, we give sufficient conditions so that the boundedness on $L^{p}$ of the Riesz transform on $M_{0}$ implies the boundedness on $L^{p}$ of the Riesz transform on $M_{1}$.
\end{abstract}

Mathematics Subject Classification (2010): 43AXX (primary); 53CXX, 58JXX (secondary).

\section{Introduction}

Let $(M, g)$ be a Riemannian manifold. The Riesz transform problem, namely giving conditions on $p$ and on the manifold such that the operator $d \Delta^{-1 / 2}$ - the so-called Riesz transform - is bounded on $L^{p}$, has recently undergone certain progress. A pioneering result which goes back to 1985 is a theorem of D. Bakry [2] which asserts that if the Ricci curvature of $M$ is non-negative, then the Riesz transform on $M$ is bounded on $L^{p}$ for every $1<p<\infty$. However, it is only recently that some progresses have been made to understand the behaviour of the Riesz transform if some amount of negative Ricci curvature is allowed. A general question is the following:

Question 1.1. What is the analogue of Bakry's result for manifolds with some (small) amount of negative Ricci curvature?

Here, the smallest of the negative part of the Ricci curvature Ric - should be understood in an integral sense, i.e. Ric $-\in L^{r}(\mathrm{~d} \mu)$, for some value of $r$ and some measure $\mathrm{d} \mu$. A partial answer has been provided by T. Coulhon and Q. Zhang in [11], where it is shown essentially that if the negative part of the Ricci curvature is smaller in an integral sense than a constant $\varepsilon$ (depending on the geometry of the manifold under consideration), then the Riesz transform is bounded on $L^{p}$ for every $1<p<\infty$. However, this result is not entirely satisfying, since it does not say what happens if the integral of the Ricci curvature is bigger than the threshold $\varepsilon$ : thus, it does not cover the case of manifolds having non-negative Ricci curvature outside a compact set. Unlike manifolds with non-negative Ricci curvature, man- 
ifolds with non-negative Ricci curvature outside a compact set can exhibit several ends, as well as more complicated topology (although it is far from being clear how to quantify this), and it has been known for already some time that Bakry's result stated as such cannot hold for manifolds with non-negative Ricci curvature outside a compact set. Indeed, as is shown by G. Carron, T. Coulhon and A. Hassell in [5], the Riesz transform on $\mathbb{R}^{n} \# \mathbb{R}^{n}$, the connected sum of two Euclidean spaces, is bounded on $L^{p}$ if and only if $1<p<n(1<p \leq 2$ if $n=2)$. In the same paper, the authors also prove that if the manifold has only one end and is isometric outside a compact set to $\mathbb{R}^{n}$, then the Riesz transform is bounded on $L^{p}$ for all $1<p<\infty$. The method of the proof - using the so-called b-calculus - was pushed further by $\mathrm{C}$. Guillarmou and A. Hassell in [17] in order to study the Riesz transform on asymptotically conical manifolds: (a particular case of) their result is that when we "glue" (that is, perform a connected sum construction) together several conical manifolds of dimension $n$, then if there is more than one end, the Riesz transform is bounded on $L^{p}$ iff $1<p<n$; and if there is only one end and the manifold is isometric outside a compact set to a conical manifold $M_{0}$, then the range of boundedness of the Riesz transform is the same as it is on $M_{0}$. The results cited above are in fact perturbation results for the Riesz transform, and one can reformulate them in the following way:

Theorem 1.2 ([5], [17]). In the class of connected asymptotically Euclidean (or more generally asymptotically conical) manifolds of dimension $n$, the boundedness of the Riesz transform on $L^{p}$ is stable:

1. Under "gluing" (that is, connected sum construction), and change of both the metric and the topology on a compact set, if $1<p<n$.

2. Under change of both the metric and the topology on a compact set, if $p \geq n$.

It is however a result very specific to the class of manifolds under consideration: the proofs rely on a precise study of the kernel of $d \Delta^{-1 / 2}$, using the difficult techniques of $b$-calculus, for which we need a precise description of the structure at infinity of both the manifold and the metric. There is thus no hope to generalize these proofs to general manifolds with non-negative Ricci curvature outside a compact set.

Then G. Carron proved in [4] a key perturbation result, which is more general. For $n>2$, let us say that a Sobolev inequality of dimension $n$ holds on $(M, g)$ if

$$
\|f\|_{\frac{2 n}{n-2}} \leq C\|\nabla f\|_{2}, \quad \forall f \in C_{0}^{\infty}(M)
$$

Let us define:

Definition 1.3. The Sobolev dimension $d_{S}(M)$ is the supremum of the set of $n$ such that the Sobolev inequality $\left(S_{n}\right)$ of dimension $n$ is satisfied on $M$ (in the case where no Sobolev inequality is satisfied on $M$, we let by convention $d_{S}=-\infty$ ).

The Sobolev dimension needs not be equal to the topological dimension of $M$, in fact if $d_{S}(M) \neq-\infty$, one has only the inequality

$$
d_{S} \geq \operatorname{dim}(M)
$$


(see [21]). For asymptotically conical manifolds, the Sobolev dimension and the topological dimension coincide, but $\mathbb{H}^{n}$, the hyperbolic space of dimension $n$, has $d_{S}\left(\mathbb{H}^{n}\right)=+\infty$. Let us introduce the following definition:

Definition 1.4. Two manifolds $M_{0}$ and $M_{1}$ are said to be isometric at infinity if there are two compact sets $K_{0}$ and $K_{1}$, of $M_{0}$ and $M_{1}$ respectively, such that $M_{0} \backslash K_{0}$ is isometric to $M_{1} \backslash K_{1}$.

Notice that by [3, Proposition 2.7], if $M_{0}$ and $M_{1}$ are isometric at infinity then $d_{S}\left(M_{0}\right)=d_{S}\left(M_{1}\right)$. Carron's perturbation result [4] states as follows:

Theorem 1.5. Let $M_{0}$ and $M_{1}$ be complete Riemannian manifolds (not necessarily connected), isometric at infinity, which satisfy $d_{S}>3$ and with Ricci curvature bounded from below. Assume that the Riesz transform on $M_{0}$ is bounded on $L^{p}$ for some $p \in\left(\frac{d_{S}}{d_{S}-1}, d_{S}\right)$. Then the Riesz transform on $M_{1}$ is bounded on $L^{p}$.

The fact that the manifolds are not supposed to be connected in this result allows one to get boundedness results for the Riesz transform when performing connected-sum constructions: for example, as a corollary, Carron recovers the fact that the Riesz transform on $\mathbb{R}^{n} \# \mathbb{R}^{n}$, the connected sum of two copies of $\mathbb{R}^{n}$, is bounded on $L^{p}$ for $1<p<n$ (under the limitation that $n>3$ ). Theorem 1.5 extends (1) of Theorem 1.2 to a much more general class of manifolds, namely to manifolds with Ricci curvature bounded from below, and satisfying a Sobolev inequality - the dimension parameter up to which we can "glue" together two such manifolds while preserving the boundedness of the Riesz transform being the Sobolev dimension $d_{S}$. Thus, we see that rather than the topological dimension, an important quantity from the point of view of the perturbation theory for the Riesz transform is the Sobolev dimension.

A way to rephrase Carron's result is that for $p<d_{S}$, the boundedness of the Riesz transform on $L^{p}$ is preserved under gluing and perturbation of both the metric and the topology on a compact set. Thus, for example, the boundedness of the Riesz transform on $L^{p}$ for any $1<p<\infty$ is preserved under gluing, perturbation of the topology and of the metric in the class of manifolds whose ends are isometric to $\mathbb{H}^{n}$ at infinity. However, when $d_{S}<\infty$, Carron's result does not say anything concerning the generalization of (2) of Theorem 1.2: explicitely, when $p \geq d_{S}$, what happens for the boundedness of the Riesz transform on $L^{p}$ if we start with a manifold with one end, and we change both the metric and the topology on a compact set, without making any gluing, i.e. preserving the fact that the manifold has only one end?

Let us mention at this point a perturbation result of Coulhon and Dungey [6] which investigates what happens for the Riesz transform if we change the metric and the Riemannian measure. Under quite mild conditions on the perturbation, they show that the boundedness on $L^{p}$ of the Riesz transform is preserved under a change of metric and of measure, for any $1<p<\infty$. However, their main assumption is that the underlying manifold is the same, that is they allow no change of topology at all, and their method relies crucially on this assumption. As a consequence, it is 
not possible, using their result, to obtain either (1) or (2) of Theorem 1.2, even for the case of the Euclidean space.

In the article [13], we used Carron's perturbation result to answer Question 1.1 for the case $p<d_{S}$ : under the assumptions that $M$ satisfies the Sobolev inequality of dimension $d>3$, that the negative part of the Ricci curvature is in $L^{\frac{d}{2}-\varepsilon} \cap L^{\infty}$, and that the volume of balls of large radius $R$ is comparable to $R^{d}$, we show that the Riesz transform is bounded on $L^{p}$ for $1<p<d$. If in addition there are no non-zero $L^{2}$ harmonic 1 -forms, we also prove that the Riesz transform is bounded on $L^{p}$ for all $1<p<\infty$. However, it is expected that this last assumption is too strong to get the boundedness on the whole $(1, \infty)$, more precisely in [13] we made the following conjecture:

Conjecture 1.6. Let $M$ satisfying the Sobolev inequality of dimension $d$, with Ric $-\in L^{\frac{d}{2}-\varepsilon} \cap L^{\infty}$ and such that the volume of balls of large radius $R$ is comparable to $R^{d}$. If $M$ has only one end, then the Riesz transform on $M$ is bounded on $L^{p}$ for every $1<p<\infty$.

In other words, is the presence of several ends the only obstruction in this class of manifolds to the boundedness of the Riesz transform on $L^{p}$ for all $1<p<\infty$ ? Motivated by this conjecture, we generalize in this article both Theorem 1.2 and Theorem 1.5. We will assume that the manifold satisfies a Sobolev inequality so that $d_{S}$, the Sobolev dimension, is greater than 2 , and we will be interested in extending the mentionned perturbation results Theorems 1.2 and 1.5 to the case where $p \geq d_{S}$. First, we define the hyperbolic dimension of $M$ to be (see Section 1)

Definition 1.7. The hyperbolic dimension $d_{H}(M)$ of $M$ is the supremum of the set of $p$ such that $M$ is $p$-hyperbolic.

Our main result shows first that $d_{H}-$ and not $d_{S}$ as Carron's result seems to indicate - is the relevant quantity to be considered when gluing is performed; and secondly, we are able to generalize (2) of Theorem 1.2 under much more general assumptions. Our result writes:

Theorem 1.8. Let $M_{0}, M_{1}$ be two Riemannian manifolds (not necessarily connected), isometric at infinity, whose Ricci curvatures are bounded from below and which satisfy $d_{S}>2$. We assume that the Riesz transform on $M_{0}$ is bounded on $L^{p}$ for $p \in\left[p_{0}, p_{1}\right)$ with

$$
\begin{cases}\frac{d_{S}}{d_{S}-1}<p_{0} \leq 2 & \text { if } d_{S}>3, \\ p_{0}=2 & \text { if } 2<d_{S} \leq 3,\end{cases}
$$

and $p_{1}>\frac{d_{S}}{d_{S}-2}$. Then the Riesz transform on $M_{1}$ is bounded on $L^{p}$ for $p \in$ $\left[p_{0}, \min \left(d_{H}(M), p_{1}\right)\right)$. If furthermore $M_{1}$ has only one end, then the Riesz transform on $M_{1}$ is bounded on $L^{p}$ for $p \in\left[p_{0}, p_{1}\right)$.

We now make a certain number of comments about this result: 


\section{Remark 1.9.}

1. We will prove in Section 1 (Proposition 2.11) that if the Riesz transform on $M$ is bounded on $L^{p}$ for $p \in\left(\frac{d_{S}}{d_{S}-1}, 2\right]$, then

$$
d_{S}(M) \leq d_{H}(M)
$$

so that under this mild assumption our result indeed generalizes Carron's result (up to endpoints of the range of boundedness). Our result says that $d_{H}$, and not $d_{S}$, is the relevant quantity to be considered when we perform a gluing. However, due to the fact that the behaviour of the Riesz transform is not known for many examples, we do not know (although we think there exists) an example of a manifold $M$ on which the Riesz transform is bounded on $L^{p}$ for $p \in\left(p_{0}, p_{1}\right)$ with $p_{1}>d_{S}$ and $d_{H}>d_{S}$. Nonetheless, we will see in Corollary 1.13 an application using $d_{H}$ and not $d_{S}$.

2. In the case where $M_{1}$ has only one end, this result extends point (2) of Theorem 1.2 to the class of manifolds satisfying a Sobolev inequality. This provides evidence in favour of Conjecture 1.6, and it could be also a necessary tool to prove it, in the same way that we used Carron's result [4] in [13] in order to prove boundedness of the Riesz transform on $L^{p}$ for $p<d_{S}$.

3. We expect that the hypothesis that $M_{0}$ satisfies a Sobolev inequality is too strong. A more reasonable hypothesis would be that $M_{0}$ satisfies the relative FaberKrahn inequality, which is equivalent (see [15]) to the fact that $M_{0}$ has the volume doubling property is doubling and that the heat kernel of the Laplacian satisfies a Gaussian upper estimate.

4. We are not able to treat the upper endpoint of the range of boundedness. However, in all the known cases (except when the interval of boundedness of the Riesz transform has 2 as an endpoint, for example $(1,2]$ in the case $\mathbb{R}^{2} \# \mathbb{R}^{2}$ ), the range of boundedness of the Riesz transform is an open interval, i.e. the Riesz transform is not bounded at the endpoints, and thus our limitation is not so disturbing. We also need to assume the technical condition $p_{1}>\frac{d_{S}}{d_{S}-2}$, which is satisfied in most cases, and in all the interesting cases covered by Carron's result, when $d_{S} \geq 4$.

5. Recall that in Carron's result, one needs to assume $d_{S}>3$. In our result, we can allow $d_{S}=3$, but in this case we can conclude only for the $p$ 's which are larger that 2. Also, we need that $p_{1}>\frac{d_{S}}{d_{S}-2}=3$, thus Theorem 1.8 gives for example that the Riesz transform on a manifold isometric at infinity to $\mathbb{R}^{3}$ is bounded on $L^{p}$ for all $2<p<\infty$, but does not tell us anything about the Riesz transform on the connected sum of two copies of $\mathbb{R}^{3}$, although we know that it is bounded on $L^{p}$ for $1<p<3$ by [5].

Theorem 1.8 has a certain number of interesting corollaries, which we describe now. The first three of them follow from Theorem (1.8) with the hypothesis " $M_{1}$ has only one end", and the last one uses the hyperbolic dimension $d_{H}$. First, we recover a particular case of a result of C. Guillarmou and A. Hassell [17] on asymptotically 
conical manifolds, without using the heavy machinery of b-calculus - as in [17], this uses H.Q. Li's result [18] about the Riesz transform on conical manifolds.

Corollary 1.10. Let $M_{1}$ be a complete Riemannian manifold, isometric at infinity to a conical manifold $M_{0}=\mathbb{R}_{+}^{\star} \times N$, with $(N, h)$ connected and compact of dimension $n-1$-that is, $M_{0}$ is endowed with the metric $g=d t^{2}+t^{2} h$. Let $\lambda_{1}$ be the first non-zero eigenvalue of the Laplacian on $N$, and let

$$
p_{0}:=\frac{n}{\frac{n}{2}-\sqrt{\lambda_{1}+\left(\frac{n-2}{2}\right)^{2}}}
$$

(with $p_{0}=\infty$ if $\lambda_{1} \geq n-1$ by convention). If $n \geq 3$, then the Riesz transform on $M_{1}$ is bounded on $L^{p}$ when $1<p<p_{0}$, and is unbounded on $L^{p}$ when $p>p_{0}$.

Furthermore, we also have the following two new results:

Corollary 1.11. Let $M$ be a complete Riemannian manifold with one end, isometric at infinity to a manifold with non-negative Ricci curvature. We assume that on $M$ the following volume estimate holds: there is $o \in M$ and $v>2$ such that

$$
V(o, R) \geq C R^{v}, \quad \forall R \geq 1,
$$

then the Riesz transform on $M$ is bounded on $L^{p}$ for all $1<p<\infty$.

Corollary 1.12. Let $M$ be isometric at infinity to a connected, simply connected nilpotent Lie group (endowed with a left-invariant Riemannian metric). Then the Riesz transform on $M$ is bounded on $L^{p}$ for every $1<p<\infty$.

Finally, we have the following corollary, which is also new:

Corollary 1.13. Let $n \geq 3$, and let $N$ be a manifold which is $q$-hyperbolic for some $q>n$, and which has Ricci curvature bounded from below. Then the Riesz transform on $M=N \# \mathbb{R}^{n}$, the connected sum of $N$ and $\mathbb{R}^{n}$, is not bounded on $L^{p}$ for $n<p<\infty$. In particular, the Riesz transform on the connected sum $\mathbb{R}^{n} \# \mathbb{H}^{n}$ of an Euclidean space and a hyperbolic space is not bounded on $L^{p}$ for any $n<p<\infty$.

The organization of this article is as follows: in Section 2, we review classical results concerning the notion of $p$-hyperbolicity, and prove some results concerning the hyperbolic dimension. In Section 3, we prove Theorem 1.8 and its corollaries.

Acknowledgements. This article is part of the $\mathrm{PhD}$ thesis of the author. The author would like to thank his advisor G. Carron, for inspiring discussions and support. 


\section{About $p$-hyperbolicity}

In this section we recall some notions concerning $p$-hyperbolicity that will be needed in the sequel. General references are [8] and [14]. We will assume that the manifold is connected. We will also assume that it is smooth, so that local elliptic theory applies. In particular, we will make use of the local Sobolev injections, of the trace theorems and of Poincaré inequalities for bounded domains. For references on this, see [21] and [23]. Let us fix $1<p<\infty$.

Definition 2.1. We say that a Riemannian manifold $(M, g)$ is p-hyperbolic if for every non-empty, relatively compact open subset $U$ of $M$, there exists a constant $C_{U}$ such that

$$
\int_{U}|f|^{p} \leq C_{U} \int_{M}|\nabla f|^{p}, \quad f \in C_{0}^{\infty}(M) .
$$

As in the case $p=2$, we have the following:

Proposition 2.2. $(M, g)$ is p-hyperbolic if and only if there exist some non-empty, relatively compact open subset $U$ of $M$ and a constant $C_{U}$ such that

$$
\int_{U}|f|^{p} \leq C_{U} \int_{M}|\nabla f|^{p}, \quad f \in C_{0}^{\infty}(M) .
$$

We write the proof for the reader's convenience.

Proof. It is enough to show that for every smooth connected open set $W$ containing $U$, there exists $C_{W}$ such that

$$
\int_{W}|f|^{p} \leq C_{W} \int_{M}|\nabla f|^{p}, \quad f \in C_{0}^{\infty}(M) .
$$

Let $V$ be a non-empty, smooth open set such that $V \subset \subset U$ and define $\Omega:=$ $W \backslash \bar{V}(\bar{V}$ being the closure of $V)$. Take $V$ so that the boundary of every connected component of $\Omega$ has non-empty intersection with $\bar{V}$. We will need the following:

Lemma 2.3. There exists a constant $C_{\Omega}$ such that

$$
\|f\|_{p} \leq C_{\Omega}\|\nabla f\|_{p}, \quad \forall f \in C_{D-N}^{\infty}(\Omega),
$$

where $C_{D-N}^{\infty}(\Omega)$ is the set of smooth functions on $\Omega$ taking value 0 on $\partial V$ (the index $D-N$ stands for "Dirichlet-Neumann").

Let us assume for a moment the result of the lemma, and let us conclude the proof of Proposition 2.2. Let $\rho$ be a smooth function whose support is included in $U$, such that $\rho \equiv 1$ on $V$. Then

$$
\|f\|_{L^{p}(W)} \leq\|\rho f\|_{L^{p}(W)}+\|(1-\rho) f\|_{L^{p}(W)} .
$$


Since $\|\rho f\|_{L^{p}(W)}=\|\rho f\|_{L^{p}(U)}$, we have by hypothesis

$$
\|\rho f\|_{L^{p}(W)} \leq C_{U}\|\nabla(\rho f)\|_{p} \leq C_{U}\left(\|f \nabla \rho\|_{p}+\|\rho \nabla f\|_{p}\right) .
$$

On the other hand, $\|\rho \nabla f\|_{p} \leq\|\rho\|_{\infty}\|\nabla f\|_{p}$, and by hypothesis, since the support of $\nabla \rho$ is contained in $U$,

$$
\|f \nabla \rho\|_{p} \leq\|\nabla \rho\|_{\infty}\|f\|_{L^{p}(U)} \leq C\|\nabla f\|_{p} .
$$

It remains to treat the term $\|(1-\rho) f\|_{L^{p}(W)}$. Thanks to Lemma 2.3, we obtain

$$
\|(1-\rho) f\|_{L^{p}(W)} \leq C\|\nabla((1-\rho) f)\|_{p} \leq C\left(\|\nabla(\rho f)\|_{p}+\|\rho\|_{\infty}\|\nabla f\|_{p}\right),
$$

and we bound as before $\|\nabla(\rho f)\|_{p}$ by $C\|\nabla f\|_{p}$.

Proof of Lemma 2.3. Working separately with each connected component of $\Omega$, we assume without loss of generality that $\Omega$ is connected. By contradiction, suppose there exists a sequence of functions $f_{n} \in C_{D-N}^{\infty}(\Omega)$ such that $\left\|f_{n}\right\|_{L^{p}}=1$, and $\left\|\nabla f_{n}\right\|_{L^{p}} \rightarrow 0$. Since $W^{1, p}(\Omega)$ is reflexive for $1<p<\infty$, up to the extraction of a subsequence we can assume that the sequence $\left(f_{n}\right)_{n \in \mathbb{N}}$ converges weakly to $f$ in $W^{1, p}(\Omega)$. But we have the compact Sobolev injection $W^{1, p}(\Omega) \hookrightarrow L^{p}$, therefore $\left(f_{n}\right)_{n \in \mathbb{N}}$ converges strongly in $L^{p}$, and as a consequence it converges strongly to $f$ in $W^{1, p}(\Omega)$. The function $f$ then satisfies $\nabla f=0$ in the weak sense, and this implies that $\nabla f=0$ strongly, hence $f$ is constant since $\Omega$ is connected. In addition, the trace theorem for $W^{1, p}$ shows that $\left.f\right|_{\partial V}=0$, and therefore $f$ is zero. This contradicts the fact that $\|f\|_{p}=1$. first:

We will also use another characterisation of $p$-hyperbolicity. Let us define

Definition 2.4. If $U$ is a non-empty, relatively compact open subset of $M$, we define its $p$-capacity by

$$
\begin{aligned}
\operatorname{Cap}_{p}(U) & =\inf \left\{\int_{M}|\nabla u|^{p}: u \in C_{0}^{\infty}(M) \text { such that }\left.u\right|_{U} \geq 1\right\} \\
& =\inf \left\{\int_{M}|\nabla u|^{p}: u \in C_{0}^{\infty}(M) \text { such that }\left.u\right|_{U} \equiv 1\right\} .
\end{aligned}
$$

The last inequality in this definition follows from the fact that the "truncation" of a function $u$ up to height 1 on $U$ decreases the energy $\int_{M}|\nabla u|^{p}$. For a detailed proof, see [14, Corollary 7.5]. With this definition, we have the following characterisation of the $p$-hyperbolicity:

Theorem 2.5. $(M, g)$ is p-hyperbolic if and only if the p-capacity of some (all) non-empty, relatively compact open set is non-zero.

For a proof, see [24, Proposition 2.1].

Remark 2.6. With the result of Theorem 2.5, it is easy to see that if $M$ is $p$ hyperbolic for some $1<p<\infty$, then $M$ has infinite volume. 
Corollary 2.7. A Riemannian manifold $(M, g)$ is p-hyperbolic if and only if one of its ends is p-hyperbolic.

Proof. It is enough to find a non-empty, relatively compact open subset $\Omega$ of $M$, whose $p$-capacity is non-zero. We take $\Omega$ such that $M \backslash \Omega=M_{1} \backslash B_{1} \sqcup \ldots \sqcup M_{k} \backslash$ $B_{k}$, the $M_{i}$ being the (closed) ends of $M$, and the $B_{i}$ being non-empty, relatively compact open subsets of $M_{i}$. Using the fact that the $p$-capacity of a non-empty, relatively compact open subset $U$ is equal to

$$
\inf \left\{\int_{M \backslash U}|\nabla u|^{p}: u \in C_{0}^{\infty} \text { such that }\left.u\right|_{U} \equiv 1\right\},
$$

we see that

$$
\operatorname{Cap}_{p}(\Omega)=\sum_{i=1}^{k} \operatorname{Cap}_{p}^{M_{i}}\left(B_{i}\right) .
$$

By hypothesis, one of the $M_{i}$ is $p$-hyperbolic ( $M_{1}$ for example), which implies $\operatorname{Cap}_{p}^{M_{1}}\left(B_{1}\right)>0$, and therefore $\operatorname{Cap}_{p}(\Omega)>0$.

The main result of this section is the following proposition concerning $p$-hyperbolicity and the Riesz transform:

Proposition 2.8. Let $(M, g)$ be a Riemannian manifold, which is p-hyperbolic for a certain $1<p<\infty$. We assume that the Riesz transform on $M$ is bounded on $L^{p}$. Then $\Delta^{-1 / 2}: L^{p} \rightarrow L_{\mathrm{loc}}^{p}$ is a bounded operator. Conversely, if the Riesz transform is bounded on $L^{q}, q$ being the dual exponent of $p$, and if

$$
\Delta^{-1 / 2}: L^{p} \rightarrow L_{\text {loc }}^{p}
$$

is a bounded operator, then $M$ is p-hyperbolic.

Proof. Recall that the domain $L^{p}$ of $\Delta^{1 / 2}$ is defined as the set of functions $h$ in $L^{p}$ such that $\frac{e^{-t \sqrt{\Delta}} h-h}{t}$ has a limit in $L^{p}$ when $t$ tends to 0 . We will first prove the following:

Lemma 2.9. For $1<p<\infty, C_{0}^{\infty}(M)$ is contained in the domain $L^{p}$ of $\Delta^{1 / 2}$, and $\Delta^{1 / 2} C_{0}^{\infty}$ is dense in $L^{p}$. Furthermore, if $u \in C_{0}^{\infty}(M)$, then $\Delta^{-1 / 2} \Delta^{1 / 2} u=u$.

Proof. If $u \in C_{0}^{\infty}(M)$, we write

$$
\Delta^{1 / 2} u=\Delta^{-1 / 2} \Delta u=\int_{0}^{\infty} e^{-t \sqrt{\Delta}} \Delta u \mathrm{~d} t
$$

and we separate the integral in $\int_{0}^{1}+\int_{1}^{\infty}=I_{1}+I_{2}$. In order to bound the $L^{p}$ norm of $I_{1}$, we use the fact that $\Delta u \in L^{p}$ and that $\left\|e^{-t \sqrt{\Delta}}\right\|_{p, p} \leq 1$, which yields

$$
\left\|I_{1}\right\|_{p} \leq\|\Delta u\|_{p}
$$


For $I_{2}$, we use the analyticity of $e^{-t \sqrt{\Delta}}$ on $L^{p}$, which implies that

$$
\left\|\Delta e^{-t \sqrt{\Delta}}\right\|_{p, p} \leq \frac{C}{t^{2}}
$$

Consequently, we obtain $\left\|I_{2}\right\|_{p} \leq C\|u\|_{p}$, which gives that $\Delta^{1 / 2} u \in L^{p}$.

Let us now show that $\Delta^{1 / 2} C_{0}^{\infty}$ is dense in $L^{p}$. First, $(\Delta+1) C_{0}^{\infty}$ is dense in $L^{p}$ : indeed, if $v \in L^{q}$ is orthogonal to $(\Delta+1) C_{0}^{\infty}$ (where $q$ is the conjugate exponent of $p$ ), then we have in the weak sense $(\Delta+1) v=0$, and this implies by a result of S.T. Yau (see [20, Theorem 4.1]) that $v$ is constant, then that $v$ is zero since $M$ is of infinite volume by Remark (2.6). So $(\Delta+1) C_{0}^{\infty}$ is dense in $L^{p}$. Then, $\Delta^{1 / 2}(\Delta+1)^{-1}$ is a bounded operator on $L^{p}$ : to see this, we write

$$
\Delta^{1 / 2}(\Delta+1)^{-1}=\int_{0}^{\infty} \Delta^{1 / 2} e^{-t(\Delta+1)} \mathrm{d} t,
$$

and we use the analyticity of $e^{-t \Delta}$ to say that

$$
\left\|\Delta^{1 / 2} e^{-t \Delta}\right\|_{p, p} \leq \frac{C}{\sqrt{t}}, \quad \forall t>0
$$

Now, we write

$$
\Delta^{1 / 2} C_{0}^{\infty}=\Delta^{1 / 2}(\Delta+1)^{-1}(\Delta+1) C_{0}^{\infty},
$$

and since $(\Delta+1) C_{0}^{\infty}$ is dense in $L^{p}$, and that $\Delta^{1 / 2}(\Delta+1)^{-1}$ is continuous on $L^{p}$, we have to see that the range of $\Delta^{1 / 2}(\Delta+1)^{-1}$ is dense in $L^{p}$. But $(\Delta+1)^{-1} L^{p}=$ $\mathcal{D}_{p}(\Delta)$, the domain $L^{p}$ of the Laplacian. So we have to see that $\Delta^{1 / 2} \mathcal{D}_{p}(\Delta)$ is dense in $L^{p}$. But $\mathcal{D}_{p}(\Delta)$ contains $\Delta^{1 / 2} C_{0}^{\infty}$ by the first part of the lemma: indeed, if $g \in C_{0}^{\infty}$,

$$
\Delta\left(\Delta^{1 / 2} g\right)=\Delta^{1 / 2}(\Delta g),
$$

and this is in $L^{p}$ since $\Delta g \in L^{p}$. Therefore $\Delta^{1 / 2} \mathcal{D}_{p}(\Delta)$ contains $\Delta^{1 / 2} \Delta^{1 / 2} C_{0}^{\infty}=$ $\Delta C_{0}^{\infty}$, which is dense in $L^{p}$ again by Yau's result.

It remains to show that when $u \in C_{0}^{\infty}$, then $\Delta^{-1 / 2} \Delta^{1 / 2} u=u$. We write

$$
\begin{aligned}
\Delta^{-1 / 2} \Delta^{1 / 2} u & =\int_{0}^{\infty} e^{-t \sqrt{\Delta}} \Delta^{1 / 2} u \mathrm{~d} t \\
& =\int_{0}^{\infty}-\frac{d}{d t}\left(e^{-t \sqrt{\Delta}} u\right) \mathrm{d} t \\
& =u-\lim _{t \rightarrow \infty} e^{-t \sqrt{\Delta}} u .
\end{aligned}
$$

By the spectral theorem, $\lim _{t \rightarrow \infty} e^{-t \sqrt{\Delta}} u$ converges in $L^{2}$ to the projection of $u$ on the $L^{2}-$ kernel of $\Delta$. But by Yau's above-mentionned result and the fact that $M$ has infinite volume, the $L^{2}-$ kernel of $\Delta$ is reduced to $\{0\}$, and therefore

$$
\Delta^{-1 / 2} \Delta^{1 / 2} u=u \text {. }
$$


Now, we come back to the proof of Proposition 2.8. We consider the first part of the proposition. Let $\Omega$ be a non-empty, open, relatively compact set in $M$. The fact that the Riesz transform is bounded on $L^{p}$ is equivalent to the inequality

$$
\|\nabla u\|_{p} \leq C\left\|\Delta^{1 / 2} u\right\|_{p}, \quad \forall u \in C_{0}^{\infty} .
$$

Since $M$ is $p$-hyperbolic, we also have the inequality

$$
\|u\|_{L^{p}(\Omega)} \leq C\|\nabla u\|_{p}, \quad \forall u \in C_{0}^{\infty} .
$$

Combining these two inequalities, we obtain

$$
\|u\|_{L^{p}(\Omega)} \leq C\left\|\Delta^{1 / 2} u\right\|_{p}, \quad \forall u \in C_{0}^{\infty} .
$$

Fix $u \in C_{0}^{\infty}$, and define $v=\Delta^{1 / 2} u$. Since $u \in C_{0}^{\infty}$, by Lemma 2.9 we have

$$
\Delta^{-1 / 2} \Delta^{1 / 2} u=u,
$$

and thus $v$ is in the $L^{p}$ domain of $\Delta^{-1 / 2}$, and moreover $\Delta^{-1 / 2} v=u$. Consequently, we obtain

$$
\left\|\Delta^{-1 / 2} v\right\|_{L^{p}(\Omega)} \leq C\|v\|_{p} .
$$

This is true for every $v \in \Delta^{1 / 2} C_{0}^{\infty}$, but by Lemma $2.9, \Delta^{1 / 2} C_{0}^{\infty}$ is dense in $L^{p}$, and thus we obtain that

$$
\left\|\Delta^{-1 / 2} v\right\|_{L^{p}(\Omega)} \leq C\|v\|_{p}, \quad \forall v \in L^{p},
$$

which is the result of the first part.

For the converse, we start with the assumption that there is a constant $C$ and a non-empty, open, relatively compact set $\Omega$ such that

$$
\left\|\Delta^{-1 / 2} v\right\|_{L^{p}(\Omega)} \leq C\|v\|_{p}, \quad \forall v \in L^{p} .
$$

Apply this to $v:=\Delta^{1 / 2} u$ for $u \in C_{0}^{\infty}(M)$ (which is licit by Lemma 2.9), and using that $\Delta^{-1 / 2} v=u$ gives

$$
\|u\|_{L^{p}(\Omega)} \leq C\left\|\Delta^{1 / 2} u\right\|_{p}, \quad \forall u \in C_{0}^{\infty}(M) .
$$

But it is well-known that the boundedness of the Riesz transform on $L^{q}$ for $\frac{1}{q}+\frac{1}{p}=$ 1, implies the following dual inequality: there is a constant $C$ such that

$$
\left\|\Delta^{1 / 2} u\right\|_{p} \leq C\|\nabla u\|_{p}, \quad \forall u \in C_{0}^{\infty}(M) .
$$

As a consequence, we get

$$
\|u\|_{L^{p}(\Omega)} \leq C\|\nabla u\|_{p}, \quad \forall u \in C_{0}^{\infty}(M),
$$

i.e. $M$ is $p$-hyperbolic. 
To conclude this section, we prove an inequality, announced in the introduction, involving the hyperbolic dimension and the Sobolev dimension. First, recall the definition given in the introduction:

Definition 2.10. The hyperbolic dimension $d_{H}$ of $M$ is defined as the supremum of the set of $p$ such that $M$ is $p$-hyperbolic.

By Corollary 2.7, the hyperbolic dimension is the supremum of the hyperbolic dimension of the ends, and this implies that if $M_{0}$ and $M_{1}$ are isometric at infinity, then

$$
d_{H}\left(M_{0}\right)=d_{H}\left(M_{1}\right) .
$$

Notice that (to the author's knowledge) it is not known in full generality that the set of $p$ such that $M$ is $p$-hyperbolic is an interval; of course, by Proposition 2.8, this is true if the Riesz transform on $M$ is bounded on $L^{p}$ for $1<p<\infty$. We have the following consequence of Proposition 2.8, announced in the introduction:

Corollary 2.11. Let $M$ satisfying $d_{S}>2$, and assume that the Riesz transform on $M$ is bounded on $L^{p}$ for $p \in\left(\frac{d_{S}}{d_{S}-1}, 2\right]$. Then

$$
d_{H} \geq d_{S} .
$$

More precisely, $M$ is p-hyperbolic for every $2 \leq p<d_{S}$.

Proof. Denote $d=d_{S}$, and let $2 \leq p<d$. By Varopoulos [25],

$$
\Delta^{-1 / 2}: L^{p} \rightarrow L^{\frac{d p}{d-p}},
$$

and in particular

$$
\Delta^{-1 / 2}: L^{p} \rightarrow L_{\mathrm{loc}}^{p} .
$$

By hypothesis, the Riesz transform on $M$ is bounded on $L^{q}, q$ being the dual of $p$, for every $2 \leq p<d$. The result follows now from Proposition 2.8.

\section{Proof of the main results}

This section is devoted to the proof of Theorem 1.8 and its corollaries, announced in the introduction. We will extend the proof of [4, Theorem 1.5], in order to get rid of the condition $p<d_{S}$. For the convenience of the reader, we have divided the proof in several subsections. First, in subsection 1, we introduce several definitions and notations. In Subsection 2, we recall the construction of [4]. In Subsection 3, we prove Theorem 1.8 in the case of several ends. In Subsection 4, we prove Theorem 1.8 in the case of one end. Finally, in Subsection 5, we prove the corollaries of Theorem 1.8. 


\subsection{Definitions and notation}

Notation 3.1. we will write $\mathfrak{s}(f)$ for the support of $f$.

Let $K_{1}$ be a compact set of $M_{1}$ with smooth boundary, such that $M_{1} \backslash K_{1}$ is isometric to the complement of a compact set of $M_{0}$, and $K_{2}, K_{3}$ compact sets of $M_{1}$ with smooth boundaries such that $K_{1} \subset K_{2} \subset K_{3}$ and such that $K_{i}$ is contained in the interior of $K_{j}$ for $i<j$.

We define $\Omega:=M_{1} \backslash K_{1}$. Let $\left(\rho_{0}, \rho_{1}\right)$ be a partition of unity such that $\left.\rho_{1}\right|_{K_{1}} \equiv$ $1, \mathfrak{s}\left(\rho_{0}\right) \subset \Omega$ and $\mathfrak{s}\left(\rho_{1}\right) \subset K_{2}$. We also take $\varphi_{0}$ and $\varphi_{1}$ two smooth functions, such that $\mathfrak{s}\left(\varphi_{0}\right) \subset \Omega, \mathfrak{s}\left(\varphi_{1}\right) \subset K_{3}$ and such that $\varphi_{i} \rho_{i}=\rho_{i}$ for $i=1,2$. Furthermore, we assume that $\left.\varphi_{1}\right|_{K_{2}} \equiv 1$.

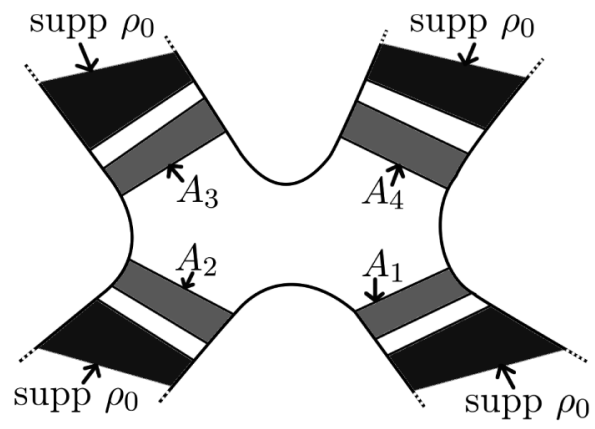

We denote by $A$ the closure of a relatively compact, smooth open subset containing $\mathfrak{s}\left(d \varphi_{0}\right)$. We can arrange so that the distance between $A$ and $\mathfrak{s}\left(\rho_{0}\right)$ is non-zero. Moreover, we can arrange so that $A$ is a disjoint union of connected "annuli" $A_{i}$, each annulus corresponding to an end of $M_{0}$.

\subsection{About Carron's proof of Theorem 1.5}

G. Carron's proof of Theorem 1.5 consists in building a parametrix for the Riesz transform: the idea is to build first a parametrix for $e^{-\sigma \sqrt{\Delta}}$; then by the formula

$$
\Delta^{-1 / 2}=\int_{0}^{\infty} e^{-\sigma \sqrt{\Delta}} \mathrm{d} \sigma
$$

the parametrix for $e^{-\sigma \sqrt{\Delta}}$ integrated in time yields a parametrix for $\Delta^{-1 / 2}$, and by differentiation in space, for the Riesz transform $d \Delta^{-1 / 2}$. Therefore Carron's proof is in two steps: first, the construction of a good parametrix for $e^{-\sigma \sqrt{\Delta}}$, such that when integrated in time and differentiated in space, it yields a parametrix bounded on $L^{p}$ for the Riesz transform. And secondly, one needs to prove that the error term between the parametrix and the Riesz transform is also bounded on $L^{p}$.

Explicitly, Carron takes for the parametrix of $e^{-\sigma \sqrt{\Delta}}$ :

$$
\mathcal{E}(\sigma, u)=\varphi_{0} e^{-\sigma \sqrt{\Delta_{0}}} \rho_{0} u+\varphi_{1} e^{-\sigma \sqrt{\Delta_{1}}} \rho_{1} u, \quad \forall u \in C_{0}^{\infty}(M),
$$


where $\Delta_{0}$ is the Laplacian on $M_{0}$, and $\Delta_{1}$ is the Laplacian on $K_{3}$ with Dirichlet boundary conditions. Here, $\rho_{0} u$ has been naturally identified to a function defined on $M_{0}$. Then we have the following formula:

$$
e^{-\sigma \sqrt{\Delta}} u=\mathcal{E}(\sigma, u)-\mathcal{G}\left[\left(-\frac{\partial^{2}}{\partial \sigma^{2}}+\Delta\right) \mathcal{E}(\sigma, u)\right],
$$

where $\mathcal{G}$ is the Green operator of $\left(-\frac{\partial^{2}}{\partial \sigma^{2}}+\Delta\right)$ on $\mathbb{R}_{+} \times M_{1}$ with Dirichlet boundary conditions on the boundary $\{0\} \times M_{1}$. Indeed, both the right and the left hand side are solutions of the Dirichlet problem

$$
\left\{\begin{array}{l}
\mathcal{L} \varphi(t, x)=0, \quad \forall(t, x) \in \mathbb{R}_{+} \times M, \\
\left.\varphi\right|_{\partial \mathbb{R}_{+} \times M}=\varphi(0, \cdot)=u
\end{array}\right.
$$

where $\mathcal{L}$ is the elliptic operator $\mathcal{L}:=-\frac{\partial}{\partial t^{2}}+\Delta$ acting on functions on $\mathbb{R}_{+} \times M$, and formula (3.1) follows by a uniqueness result for the solution of the Dirichlet problem (for more details, see the related proof of [13, Proposition 5.2.1 ]). The term $\mathcal{G}\left[\left(-\frac{\partial^{2}}{\partial \sigma^{2}}+\Delta\right) \mathcal{E}(\sigma, u)\right]$ is the error term in the parametrix of $e^{-\sigma \sqrt{\Delta}}$. When integrated and differentiated, the above parametrix for $e^{-\sigma \sqrt{\Delta}}$ yields a parametrix for the Riesz transform, which is explicitely

$$
\mathcal{R}:=\sum_{i=0}^{1} \varphi_{i} d \Delta_{i}^{-1 / 2} \rho_{i}+\left(d \varphi_{i}\right) \Delta_{i}^{-1 / 2} \rho_{i} .
$$

Let us explain why $\mathcal{R}$ is a good parametrix for $p<d_{S}$, i.e. is bounded on $L^{p}$ if $p<d_{S}$. First, $d \Delta_{0}^{-1 / 2}$ is the Riesz transform on $M_{0}$, which is bounded by hypothesis. Also, $\varphi_{1} d \Delta_{1}^{-1 / 2} \rho_{1}$ is a pseudo-differential operator with compact support, and hence is bounded on $L^{p} ;\left(d \varphi_{0}\right) \Delta_{0}^{-1 / 2} \rho_{0}$ is an operator with smooth kernel and compact support, hence is bounded on $L^{p}$. Finally, the operator $\left(d \varphi_{0}\right) \Delta_{0}^{-1 / 2} \rho_{0}$ is bounded on $L^{p}$ if $p<d_{S}$, which comes from the facts that $d \varphi_{0}$ is compactly supported and that for $p<d_{S}$,

$$
\Delta_{0}^{-1 / 2}: L^{p} \rightarrow L^{\frac{n p}{n-p}} .
$$

The second part of Carron's proof is to show that the error term when we approximate $d \Delta^{-1 / 2}$ by $\mathcal{R}$ can be controled on $L^{p}$ if $p<d_{S}$.

In order to improve Carron's result, two things have to be done: first, to find a parametrix for the Riesz transform which is bounded on $L^{p}$ for $p \geq d_{S}$, and secondly, to improve the estimates of the error term in order to show that it is bounded on $L^{p}$ for $p \geq d_{S}$, and not only for $p<d_{S}$. 


\subsection{The case where $M$ has several ends}

In this subsection, we prove Theorem 1.8 in the case where $M_{1}$ has several ends. We first remark that under our hypotheses, the boundedness of the Riesz transform of $M_{1}$ on $L^{p}$ for $p \in\left[p_{0}, 2\right]$ is a consequence of Carron's work [4] and we do not improve it. We will only prove boundedness in the range $\left[2, \min \left(d_{H}, p_{1}\right)\right)$. We take the same parametrix for $e^{-\sigma \sqrt{\Delta}}$ as in Carron [4]:

$$
\mathcal{E}(\sigma, u)=\varphi_{0} e^{-\sigma \sqrt{\Delta_{0}}} \rho_{0} u+\varphi_{1} e^{-\sigma \sqrt{\Delta_{1}}} \rho_{1} u .
$$

The main observation is that when $p \in\left[2, \min \left(p_{1}, d_{H}\right)\right)$, the corresponding parametrix for the Riesz transform $\mathcal{R}=d \int_{0}^{\infty} \mathcal{E}(\sigma, \cdot) \mathrm{d} \sigma$ is bounded on $L^{p}$. Let us explain this now. We have seen in the previous paragraph that

$$
\mathcal{R}:=\sum_{i=0}^{1} \varphi_{i} d \Delta_{i}^{-1 / 2} \rho_{i}+\left(d \varphi_{i}\right) \Delta_{i}^{-1 / 2} \rho_{i},
$$

and that under the hypothesis of Theorem 1.8, the operators $\varphi_{0} d \Delta_{0}^{-1 / 2} \rho_{0}$, $\varphi_{1} d \Delta_{1}^{-1 / 2} \rho_{1}$ and $\left(d \varphi_{1}\right) \Delta_{1}^{-1 / 2} \rho_{1}$ are bounded on $L^{p}$ for $p \in\left(p_{0}, p_{1}\right)$. It remains the operator $\left(d \varphi_{0}\right) \Delta_{0}^{-1 / 2} \rho_{0}$. By the fact that $M_{0}$ satisfies the Sobolev inequality, $M_{0}$ is 2 -hyperbolic. Thus by the result of Proposition 2.8 and interpolation, $\left(d \varphi_{1}\right) \Delta_{1}^{-1 / 2} \rho_{1}$ is bounded on $L^{p}$ if $p \in\left[2, d_{H}\right)$. Therefore, $\mathcal{R}$ is bounded for every $p \in\left[2, \min \left(p_{1}, d_{H}\right)\right)$. All that remains to be done is to show that the corresponding error term is bounded on $L^{p}$ when $p \in\left[2, \min \left(p_{1}, d_{H}\right)\right)$, and for this we need to improve the error estimates of [4].

Let $p \in\left[2, \min \left(p_{1}, d_{H}\right)\right)$; we choose some fixed $q>\frac{d_{S}}{d_{S}-2}$ satisfying $p<$ $q<\min \left(p_{1}, d_{H}\right)$. We will also take $d$ close enough to $d_{S}$, such that the Sobolev inequality of dimension $d$ is satisfied on $M$. We will choose $d$ later, depending on $p$. According to [4], the error term in the parametrix of the Riesz transform is $d g$, where

$$
g=\int_{0}^{\infty} \int_{\mathbb{R}_{+} \times R_{+} \times M} \mathcal{G}(\sigma, s, x, y)\left[\left(-\frac{\partial^{2}}{\partial \sigma^{2}}+\Delta\right) \mathcal{E}(\cdot, u)(s, y)\right] \mathrm{d} \sigma \mathrm{d} s \mathrm{~d} y,
$$

$\mathcal{G}$ being the Green function of $\left(-\frac{\partial^{2}}{\partial t^{2}}+\Delta\right)$ on $M \times \mathbb{R}_{+}$with Dirichlet boundary conditions on $M \times\{0\}$. We let

$$
\left(-\frac{\partial^{2}}{\partial \sigma^{2}}+\Delta\right) \mathcal{E}(\sigma, u)=f_{0}(\sigma, .)+f_{1}(\sigma, .),
$$

where the functions $f_{i}$ are defined by

$$
f_{i}(\sigma, .)=\left(\Delta \varphi_{i}\right)\left(e^{-\sigma \sqrt{\Delta_{i}}} \rho_{i} u\right)-2\left\langle d \varphi_{i}, \nabla e^{-\sigma \sqrt{\Delta_{i}}} \rho_{i} u\right\rangle .
$$


In [4], estimates on the $f_{i}$ are shown. However, since we do not assume $p<d_{S}$, the corresponding estimates for $f_{0}$ will not hold in our case. Instead, we will estimate a modified function $\tilde{f}_{0}$, that we define by

$$
\tilde{f}_{0}(\sigma, .)=\left[\sum_{j} \mathbf{1}_{A_{j}}\left(\Delta \varphi_{0}\right)\left(e^{-\sigma \sqrt{\Delta_{0}}} \rho_{0} u-\left(e^{-\sigma \sqrt{\Delta_{0}}} \rho_{0} u\right)_{A_{j}}\right)\right]-2\left\langle d \varphi_{0}, \nabla e^{-\sigma \sqrt{\Delta_{0}}} \rho_{0} u\right\rangle,
$$

where $A=\sqcup_{j} A_{j}$, each $A_{j}$ being connected and smooth (see subsection 1 for the definition of $A$ ), and $\left(e^{-\sigma \sqrt{\Delta_{0}}} \rho_{0} u\right)_{A_{j}}$ denotes the average of $e^{-\sigma \sqrt{\Delta_{0}}} \rho_{0} u$ on $A_{j}$. We first show estimates on $f_{1}$ and $\tilde{f}_{0}$ :

Lemma 3.2. If $\alpha=d\left(\frac{1}{p}-\frac{1}{q}\right)>0$, then there exists a constant $C$ independent of $u$ such that

$$
\left\|\tilde{f}_{0}(\sigma, .)\right\|_{1}+\left\|\tilde{f}_{0}(\sigma, .)\right\|_{p} \leq \frac{C}{(1+\sigma)^{1+\alpha}}\|u\|_{p}, \quad \forall \sigma>0
$$

and

$$
\left\|f_{1}(\sigma, .)\right\|_{1}+\left\|f_{1}(\sigma, .)\right\|_{p} \leq \frac{C}{(1+\sigma)^{1+\alpha}}\|u\|_{p}, \quad \forall \sigma>0 .
$$

Proof of Lemma 3.2. We begin with $f_{1}$. In [4], it is shown that for some constant $\lambda>0$,

$$
\left\|f_{1}(\sigma, .)\right\|_{1}+\left\|f_{1}(\sigma, .)\right\|_{p} \leq e^{-\lambda \sigma}\|u\|_{p}, \quad \forall \sigma>0,
$$

which of course implies

$$
\left\|f_{1}(\sigma, .)\right\|_{1}+\left\|f_{1}(\sigma, .)\right\|_{p} \leq \frac{C}{(1+\sigma)^{1+\alpha}}\|u\|_{p}, \quad \forall \sigma>0 .
$$

Now we turn to $\tilde{f}_{0}$. Since $d \Delta_{0}^{-1 / 2}$ is bounded on $L^{q}\left(M_{0}\right)$, and $e^{-\sigma \sqrt{\Delta_{0}}}$ is analytic on $L^{r}$ for $1<r<\infty$ (see [22] or [10], this comes from the subordination identity), we have

$$
\left\|\nabla e^{-\sigma \sqrt{\Delta_{0}}}\right\|_{q, q} \leq \frac{C}{\sigma}, \quad \forall \sigma>0 .
$$

Also,

$$
\left\|e^{-\sigma \sqrt{\Delta_{0}}}\right\|_{p, q} \leq \frac{C}{\sigma^{d\left(\frac{1}{p}-\frac{1}{q}\right)}}=\frac{C}{\sigma^{\alpha}}, \quad \forall \sigma>0 .
$$

We get in particular

$$
\left\|\nabla e^{-\sigma \sqrt{\Delta_{0}}}\right\|_{p, q} \leq\left\|\nabla e^{-\frac{\sigma}{2} \sqrt{\Delta_{0}}}\right\|_{q, q}\left\|e^{-\frac{\sigma}{2} \sqrt{\Delta_{0}}}\right\|_{p, q} \leq \frac{C}{\sigma^{1+\alpha}}, \quad \forall \sigma \geq 1 .
$$

We also have (cf. [4])

$$
\left\|\nabla e^{-\sigma \sqrt{\Delta_{0}}}\right\|_{L^{p}(U) \rightarrow L^{q}(F)} \leq C, \quad \forall \sigma \leq 1,
$$


if $U$ is an open subset and $F$ a compact set at positive distance from $U$. Therefore we get

$$
\left\|\nabla e^{-\sigma \sqrt{\Delta_{0}}}\right\|_{L^{p}(U) \rightarrow L^{q}(F)} \leq \frac{C}{(1+\sigma)^{1+\alpha}}, \quad \forall \sigma>0 .
$$

Using the fact that for every compact $F, L^{q}(F) \hookrightarrow L^{1}(F)$ and $L^{q}(F) \hookrightarrow L^{p}(F)$, and given that the support of $\rho_{0}$ and $A$ are disjoint, we obtain

$$
\begin{array}{r}
\left\|\left\langle d \varphi_{0}, \nabla e^{-\sigma \sqrt{\Delta_{0}}} \rho_{0} u\right\rangle\right\|_{L^{1}}+\left\|\left\langle d \varphi_{0}, \nabla e^{-\sigma \sqrt{\Delta_{0}}} \rho_{0} u\right\rangle\right\|_{L^{p}} \leq \frac{C}{(1+\sigma)^{1+\alpha}}\|u\|_{p}, \\
\forall \sigma>0 .
\end{array}
$$

It remains the term $\left[\sum_{j} \mathbf{1}_{A_{j}}\left(\Delta \varphi_{0}\right)\left(e^{-\sigma \sqrt{\Delta_{0}}} \rho_{0} u-\left(e^{-\sigma \sqrt{\Delta_{0}}} \rho_{0} u\right)_{A_{j}}\right)\right]$. We have, by the $L^{q}$-Poincaré inequality on each $A_{j}$ :

$$
\begin{aligned}
\left\|\sum_{j} \mathbf{1}_{A_{j}}\left(\Delta \varphi_{0}\right)\left(e^{-\sigma \sqrt{\Delta_{0}}} \rho_{0} u-\left(e^{-\sigma \sqrt{\Delta_{0}}} \rho_{0} u\right)_{A_{j}}\right)\right\|_{L^{q}\left(A_{j}\right)} & \leq C\left\|\nabla e^{-\sigma \sqrt{\Delta_{0}}} \rho_{0} u\right\|_{L^{q}\left(A_{j}\right)} \\
& \leq \frac{C}{(1+\sigma)^{1+\alpha}}\|u\|_{p} .
\end{aligned}
$$

Hence the estimates for $\tilde{f}_{0}$.

Now, we decompose $g$ into $g_{1}+g_{2}$, with

$$
\begin{aligned}
g_{1}(x)= & \int_{\mathbb{R}_{+} \times \mathbb{R}_{+} \times M} \mathcal{G}(\sigma, s, x, y) \tilde{f}_{0}(s, y) \mathrm{d} \sigma \mathrm{d} s \mathrm{~d} y \\
& +\int_{\mathbb{R}_{+} \times \mathbb{R}_{+} \times M} \mathcal{G}(\sigma, s, x, y) f_{1}(s, y) \mathrm{d} \sigma \mathrm{d} s \mathrm{~d} y,
\end{aligned}
$$

and

$$
g_{2}(x)=\sum_{j} \int_{\mathbb{R}_{+} \times \mathbb{R}_{+} \times M} \mathcal{G}(\sigma, s, x, y) \mathbf{1}_{A_{j}}(y)\left(\Delta \varphi_{0}\right)(y)\left(e^{-s \sqrt{\Delta_{0}}} \rho_{0} u\right)_{A_{j}} \mathrm{~d} \sigma \mathrm{d} s \mathrm{~d} y .
$$

We have, in an equivalent way ( $c f .[4])$,

$$
g_{1}=\frac{2}{\sqrt{\pi}} \int_{\mathbb{R}_{+} \times \mathbb{R}_{+}} e^{-r^{2}}\left(\int_{0}^{\frac{s^{2}}{4 r^{2}}} e^{-t \Delta}\left(\tilde{f}_{0}(s, .)+f_{1}(s, .)\right) \mathrm{d} t\right) \mathrm{d} r \mathrm{~d} s,
$$

and

$$
g_{2}=\sum_{j} \frac{2}{\sqrt{\pi}} \int_{\mathbb{R}_{+} \times \mathbb{R}_{+}} e^{-r^{2}}\left(\int_{0}^{\frac{s^{2}}{4 r^{2}}} e^{-t \Delta}\left(\mathbf{1}_{A_{j}}\left(\Delta \varphi_{0}\right)\left(e^{-s \sqrt{\Delta_{0}}} \rho_{0} u\right)_{A_{j}}\right) \mathrm{d} t\right) \mathrm{d} r \mathrm{~d} s .
$$


In order to conclude the proof of Theorem 1.8 in the case of several ends, we have to show that $\left\|d g_{1}\right\|_{p}+\left\|d g_{2}\right\|_{p} \leq C\|u\|_{p}$. This will be done in the next two lemmas. Let us begin with:

Lemma 3.3. There exists a constant $C$ such that for every $u \in L^{p}$,

$$
\left\|d g_{1}\right\|_{p} \leq C\|u\|_{p} .
$$

Proof. According to [4, Proposition 2.1], it is enough to show that $\left\|g_{1}\right\|_{p}+\left\|\Delta g_{1}\right\|_{p} \leq$ $C\|u\|_{p}$. The term $\left\|\Delta g_{1}\right\|_{p}$ is the easiest: defining $h:=\tilde{f}_{0}+f_{1}$, we have

$$
\begin{aligned}
\Delta g_{1} & =\frac{2}{\sqrt{\pi}} \int_{\mathbb{R}_{+} \times \mathbb{R}_{+}} e^{-r^{2}}\left(\int_{0}^{\frac{s^{2}}{4 r^{2}}} \Delta\left(e^{-t \Delta} h(s, .)\right) \mathrm{d} t\right) \mathrm{d} r \mathrm{~d} s \\
& =-\frac{2}{\sqrt{\pi}} \int_{\mathbb{R}_{+} \times \mathbb{R}_{+}} e^{-r^{2}}\left(\int_{0}^{\frac{s^{2}}{4 r^{2}}} \frac{d}{d t}\left(e^{-t \Delta} h(s, .)\right) \mathrm{d} t\right) \mathrm{d} r \mathrm{~d} s \\
& =\frac{2}{\sqrt{\pi}} \int_{\mathbb{R}_{+} \times \mathbb{R}_{+}} e^{-r^{2}}\left(h(s, .)-e^{-\frac{s^{2}}{4 r^{2}}} h(s, .)\right) \mathrm{d} r \mathrm{~d} s .
\end{aligned}
$$

Hence, by (3.2) and (3.3),

$$
\begin{aligned}
\left\|\Delta g_{1}\right\|_{p} & \leq \frac{4}{\sqrt{\pi}} \int_{\mathbb{R}_{+} \times \mathbb{R}_{+}} e^{-r^{2}}\|h(s, .)\|_{p} \mathrm{~d} r \mathrm{~d} s \\
& \leq \frac{4}{\sqrt{\pi}}\left(\int_{\mathbb{R}_{+} \times \mathbb{R}_{+}} e^{-r^{2}} \frac{C}{(1+s)^{1+\alpha}} \mathrm{d} r \mathrm{~d} s\right)\|u\|_{p} \\
& \leq C\|u\|_{p} .
\end{aligned}
$$

For $\left\|g_{1}\right\|_{p}$, using

$$
\left\|e^{-t \Delta}\right\|_{1, p} \leq \frac{C}{t^{\frac{d}{2}\left(1-\frac{1}{p}\right)}},
$$

and (3.2), (3.3), we have

$$
\begin{aligned}
\left\|g_{1}\right\|_{p} & \leq \frac{2}{\sqrt{\pi}} \int_{\mathbb{R}_{+} \times \mathbb{R}_{+}} e^{-r^{2}}\left(\int_{0}^{\frac{s^{2}}{4 r^{2}}}\left\|e^{-t \Delta} h(s, .)\right\|_{p} \mathrm{~d} t\right) \mathrm{d} s \mathrm{~d} r \\
& \leq \frac{2}{\sqrt{\pi}}\left(\int_{\mathbb{R}_{+} \times \mathbb{R}_{+}} e^{-r^{2}}\left(\int_{0}^{\frac{s^{2}}{4 r^{2}}} \frac{C}{\max \left(1, t^{\frac{d}{2}\left(1-\frac{1}{p}\right)}\right)(1+s)^{1+\alpha}} \mathrm{d} t\right) \mathrm{d} s \mathrm{~d} r\right)\|u\|_{p} \\
& \leq C\left(\int_{\mathbb{R}_{+} \times \mathbb{R}_{+}} e^{-r^{2}} \frac{1}{\max \left(1, t^{\frac{d}{2}\left(1-\frac{1}{p}\right)}\right)(1+2 r \sqrt{t})^{\alpha}} \mathrm{d} t \mathrm{~d} r\right)\|u\|_{p} .
\end{aligned}
$$


We separate the integral in $\int_{t \leq r^{-2}}+\int_{t \geq r^{-2}}=I_{1}+I_{2}$. The integral $I_{1}$ is finite if and only if

$$
(-2)\left(\frac{d}{2}\left(1-\frac{1}{p}\right)-1\right)<1 \text {, }
$$

which is equivalent to

$$
p>\frac{d}{d-1} .
$$

Since $p>p_{0}>\frac{d_{S}}{d_{S}-1}$, we can choose $d$ close enough to $d_{S}$ so that the inequality $p>\frac{d}{d-1}$ is satisfied. For $I_{2}$,

$$
\begin{aligned}
I_{2} & \leq \int_{0}^{\infty} e^{-r^{2}}\left(\int_{r^{-2}}^{\infty} \frac{1}{t^{\frac{d}{2}\left(1-\frac{1}{p}\right)}} \frac{1}{(r \sqrt{t})^{\alpha}} d t\right) d r \\
& \leq \int_{0}^{\infty} e^{-r^{2}} \frac{1}{r^{\alpha}}\left(\int_{r^{-2}}^{\infty} \frac{1}{t^{\frac{d}{2}\left(1-\frac{1}{p}\right)}} \frac{1}{(\sqrt{t})^{\alpha}} \mathrm{d} t\right) \mathrm{d} r .
\end{aligned}
$$

The integral in $t$ is finite if and only if

$$
\frac{d}{2}\left(1-\frac{1}{p}\right)+\frac{\alpha}{2}>1
$$

and recalling that $\alpha=d\left(\frac{1}{p}-\frac{1}{q}\right)$, we find that it is equivalent to

$$
q>\frac{d}{d-2} .
$$

Once again, since we assumed $q>\frac{d_{S}}{d_{S}-2}$, we can choose $d$ close enough to $d_{S}$ so that $q>\frac{d}{d-2}$ is satisfied. The integral in $r$ is then

$$
\int_{0}^{\infty} e^{-r^{2}} \frac{1}{r^{\alpha-2\left(\frac{d}{2}\left(1-\frac{1}{p}\right)+\frac{\alpha}{2}-1\right)}} \mathrm{d} r
$$

which is finite if and only if

$$
\alpha-d\left(1-\frac{1}{p}\right)-\alpha+2<1
$$

which is equivalent to

$$
p>\frac{d}{d-1},
$$

which is satisfied by one of our previous assumptions on $d$. 
Now we turn to estimate $d g_{2}$, which will conclude the proof of Theorem 1.8 in the case of several ends.

\section{Lemma 3.4.}

$$
\left\|d g_{2}\right\|_{p} \leq C\|u\|_{p}
$$

Proof. According to [4, Proposition 2.1], it is enough to show that $\left\|g_{2}\right\|_{p}+$ $\left\|\Delta g_{2}\right\|_{p} \leq C\|u\|_{p}$. We begin to show that $\left\|g_{2}\right\|_{p} \leq C\|u\|_{p}$. We have

$$
\begin{aligned}
g_{2}(x) x & =\sum_{j} \frac{2}{\sqrt{\pi}} \int_{\mathbb{R}_{+} \times \mathbb{R}_{+}} e^{-r^{2}}\left(\int_{0}^{\frac{s^{2}}{4 r^{2}}} e^{-t \Delta}\left(\mathbf{1}_{A_{j}}\left(\Delta \varphi_{0}\right)\left(e^{-s \sqrt{\Delta_{0}}} \rho_{0} u\right)_{A_{j}}\right)(x) \mathrm{d} t\right) \mathrm{d} r \mathrm{~d} s \\
& =\sum_{j} \frac{2}{\sqrt{\pi}} \int_{\mathbb{R}_{+} \times \mathbb{R}_{+}} e^{-r^{2}}\left(\int_{0}^{\frac{s^{2}}{4 r^{2}}}\left(e^{-s \sqrt{\Delta_{0}}} \rho_{0} u\right)_{A_{j}} e^{-t \Delta}\left(\mathbf{1}_{A_{j}} \Delta \varphi_{0}\right)(x) \mathrm{d} t\right) \mathrm{d} r \mathrm{~d} s,
\end{aligned}
$$

therefore

$$
\left\|g_{2}\right\|_{p} \leq \sum_{j} \frac{2}{\sqrt{\pi}} \int_{\mathbb{R}_{+} \times \mathbb{R}_{+}} e^{-r^{2}}\left(\int_{0}^{\frac{s^{2}}{4 r^{2}}}\left(\frac{1}{\left|A_{j}\right|} \int_{A_{j}} e^{-s \sqrt{\Delta_{0}}}\left|\rho_{0} u\right|\right)\left\|e^{-t \Delta} \chi\right\|_{p} \mathrm{~d} t\right) \mathrm{d} r \mathrm{~d} s,
$$

where we have defined $\chi:=\Delta \varphi_{0}=\Delta\left(\varphi_{0}-1\right)$. Using the fact that $\left\|e^{-t \Delta}\right\|_{1, p} \leq$ $\frac{C}{t^{\frac{d}{2}\left(1-\frac{1}{p}\right)}}$, the analyticity of $e^{-t \Delta}$ on $L^{p}$, and the fact that $\varphi_{0}-1$ is smooth with compact support,

$$
\left\|e^{-t \Delta} \chi\right\|_{p} \leq \frac{C}{\max \left(1, t^{1+\frac{d}{2}\left(1-\frac{1}{p}\right)}\right)}, \forall t>0 .
$$

Furthermore, we have for every $p>1$,

$$
1+\frac{d}{2}\left(1-\frac{1}{p}\right)>1
$$

and consequently

$$
\int_{0}^{\infty}\left\|e^{-t \Delta} \chi\right\|_{p} \mathrm{~d} t<\infty
$$

So

$$
\begin{aligned}
\left\|g_{2}\right\|_{p} & \leq C \sum_{j} \int_{\mathbb{R}_{+} \times \mathbb{R}_{+}} e^{-r^{2}}\left(\int_{A_{j}} e^{-s \sqrt{\Delta_{0}}}\left|\rho_{0} u\right|\right) \mathrm{d} r \mathrm{~d} s \\
& \leq C \sum_{j} \int_{A_{j}}\left(\int_{0}^{\infty} e^{-s \sqrt{\Delta_{0}}}\left|\rho_{0} u\right| \mathrm{d} s\right) \\
& \leq C \sum_{j} \int_{A_{j}} \Delta_{0}^{-1 / 2}\left|\rho_{0} u\right| .
\end{aligned}
$$


According to Proposition 2.8, $\Delta_{0}^{-1 / 2}: L^{p} \rightarrow L_{\mathrm{loc}}^{p} \hookrightarrow L_{\mathrm{loc}}^{1}$, which implies that

$$
\left\|g_{2}\right\|_{p} \leq C\|u\|_{p}
$$

Let us turn now to $\Delta g_{2}$ : as for $g_{1}$, we have

$$
\begin{aligned}
\left\|g_{2}\right\|_{p} & \leq \sum_{j} \frac{4}{\pi} \int_{\mathbb{R}_{+}^{2}} e^{-r^{2}}\left\|\left(e^{-s \sqrt{\Delta_{0}}} \rho_{0} u\right)_{A_{j}}\left(\Delta \varphi_{0}\right)\right\|_{p} \mathrm{~d} r \mathrm{~d} s \\
& \leq C \sum_{j} \int_{0}^{\infty}\left|\left(e^{-s \sqrt{\Delta_{0}}} \rho_{0} u\right)_{A_{j}}\right| \mathrm{d} s,
\end{aligned}
$$

and by the argument already used,

$$
\sum_{j} \int_{0}^{\infty}\left|\left(e^{-s \sqrt{\Delta_{0}}} \rho_{0} u\right)_{A_{j}}\right| \mathrm{d} s \leq C\|u\|_{p},
$$

which concludes the proof.

\subsection{The case where $M$ has one end}

In this subsection, we prove Theorem 1.8 in the case where $M_{1}$ has only one end. As we have already explained, the parametrix $\mathcal{R}$ for the Riesz transform constructed by Carron has a term which is unbounded on $L^{p}$ when $p>d_{H}$ : more precisely, the term $(d \varphi) \Delta_{0}^{-1 / 2} \rho_{0}$ is unbounded on $L^{p}$ if $p>d_{H}$. Hence, we have to modify the parametrix. The main idea is the following: notice that since $M_{1}$ has only one end, $d \varphi$ is the supported in $A$ which is a connected annulus. Since $A$ is connected and smooth, the $L^{p}$ Poincare inequality in $A$ holds, i.e. there is a constant $C$ such that

$$
\left\|v-\frac{1}{|A|} \int_{A} v\right\|_{L^{p}(A)} \leq C\|\nabla v\|_{p}, \quad \forall v \in C^{\infty}(A) .
$$

Applying this to $\Delta_{0}^{-1 / 2} \rho_{0} u$ for $u \in C_{0}^{\infty}(M)$, we get for $p \in\left[2, p_{1}\right)$

$$
\left\|\Delta_{0}^{-1 / 2} \rho_{0} u-\frac{1}{|A|} \int_{A} \Delta_{0}^{1 / 2} \rho_{0} u\right\|_{L^{p}(A)} \leq C\left\|\nabla \Delta_{0}^{-1 / 2} \rho_{0} u\right\|_{p} \leq C\|u\|_{p},
$$

where in the last inequality we have used the fact that the Riesz transform on $M_{0}$ is bounded on $L^{p}$ if $p \in\left[2,, p_{1}\right)$. This implies that the modified parametrix

$$
\mathcal{F} u=\sum_{i=0}^{1} \varphi_{i} d \Delta_{i}^{-1 / 2} \rho_{i} u+\left(d \varphi_{1}\right) \Delta_{1}^{-1 / 2} \rho_{1} u+\left(d \varphi_{0}\right)\left(\Delta^{-1 / 2} \rho_{0} u-\left(\frac{1}{|A|} \int_{A} \Delta_{0}^{-1 / 2} \rho_{0} u\right)\right)
$$


is bounded on $L^{p}$ for every $p \in\left[2, p_{1}\right)$. The corresponding parametrix for $e^{-\sigma \sqrt{\Delta}}$ is given by

$$
\mathcal{S}(\sigma, u)=\mathcal{E}(\sigma, u)-\left(\varphi_{0}-1\right)\left(\frac{1}{|A|} \int_{A} e^{-\sigma \sqrt{\Delta_{0}}} \rho_{0} u\right),
$$

i.e., there holds

$$
\mathcal{F} u=d \int_{0}^{\infty} \mathcal{S}(\sigma, u) \mathrm{d} \sigma
$$

The supplementary term that we have added to the parametrix of $e^{-\sigma \sqrt{\Delta}}$ is

$$
-\left(\varphi_{0}-1\right)\left(\frac{1}{|A|} \int_{A} e^{-\sigma \sqrt{\Delta_{0}}} \rho_{0} u\right),
$$

which vanishes when $\sigma=0$, since $A$ and the support of $\rho_{0}$ are disjoint by hypothesis. So we have, as should be,

$$
\mathcal{S}(0, u)=u
$$

Notice also that since $\varphi_{0}-1$ is compactly supported, the integral with respect to $\sigma$ of this supplementary term is analogous to the term $G_{3}$ in the parametrix of $\Delta^{-1 / 2}$ constructed by Carron-Coulhon-Hassell in [5]: its kernel $k(x, y)$ is non-zero only if $x$ is in $K_{3}$ and $y$ is in $M_{1} \backslash K_{1}$.

Thus, we have constructed a parametrix $\mathcal{F}$ for the Riesz transform, which is bounded on $L^{p}$ for $p \in\left[2, p_{1}\right)$. As in the proof of Theorem 1.8 in the case where $M_{1}$ has several ends, it remains to show that the error term is also bounded on $L^{p}$.

We will use the calculations made in the previous subsection. This time, we have (with $f_{1}$ and $\tilde{f}_{0}$ defined as in the previous subsection)

$$
\left(-\frac{\partial^{2}}{\partial \sigma^{2}}+\Delta\right) \mathcal{S}(\sigma, u)=f_{1}(\sigma, .)+\tilde{f}_{0}(\sigma, \cdot)-\left(\varphi_{0}-1\right)\left(\frac{1}{|A|} \int_{A} \Delta_{0} e^{-\sigma \sqrt{\Delta_{0}}} \rho_{0} u\right) \text {. }
$$

Define

$$
\bar{f}_{0}(\sigma, .)=\left(\varphi_{0}-1\right)\left(\frac{1}{|A|} \int_{A} \Delta_{0} e^{-\sigma \sqrt{\Delta_{0}}} \rho_{0} u\right) .
$$

We have the following estimates on $f_{1}, \tilde{f}_{0}$ and $\bar{f}_{0}$ :

Lemma 3.5. If $\alpha=d\left(\frac{1}{p}-\frac{1}{q}\right)$, then for all $\sigma>0$,

$$
\begin{aligned}
\left\|f_{1}(\sigma, \cdot)\right\|_{1}+\left\|f_{1}(\sigma, \cdot)\right\|_{p} & \leq \frac{C}{(1+\sigma)^{1+\alpha}}\|u\|_{p}, \\
\left\|\tilde{f}_{0}(\sigma, \cdot)\right\|\left\|_{1}+\right\| \tilde{f}_{0}(\sigma, \cdot) \|_{p} & \leq \frac{C}{(1+\sigma)^{1+\alpha}}\|u\|_{p},
\end{aligned}
$$


and

$$
\left\|\bar{f}_{0}(\sigma, \cdot)\right\|_{1}+\left\|\bar{f}_{0}(\sigma, \cdot)\right\|_{p} \leq \frac{C}{(1+\sigma)^{2}}\|u\|_{p}
$$

Once this lemma is established, the estimate of the error term proceeds as in the proof of Theorem 1.8 in the case where $M$ has more than one end. All we have to do is thus to prove the above estimates.

Proof of Lemma 3.5. We already proved the estimates on $f_{1}$ and $\tilde{f}_{0}$ in Lemma 3.2. It remains to treat $\bar{f}_{0}$. First, by analyticity of $e^{-\sigma \sqrt{\Delta_{0}}}$,

$$
\left\|\Delta_{0} e^{-\sigma \sqrt{\Delta_{0}}}\right\|_{p, p} \leq \frac{C}{\sigma^{2}}
$$

and therefore, using the fact that $\bar{f}_{0}(\sigma, \cdot)$ has compact support independent of $u$,

$$
\left\|\bar{f}_{0}(\sigma, \cdot)\right\|_{1,1}+\left\|\bar{f}_{0}(\sigma, \cdot)\right\|_{p, p} \leq \frac{C}{\sigma^{2}}
$$

The proof will be complete once we show that $\Delta_{0} e^{-\sigma \sqrt{\Delta_{0}}}$ is bounded $L^{p}\left(M_{0} \backslash\right.$ $\left.A_{\delta}\right) \rightarrow L^{\infty}(A)$ when $\sigma \rightarrow 0$ (where $\delta$ is a strictly positive constant, and where $A_{\delta}$ is the set of points whose distance to $A$ is less than $\delta$ ). For this, we use the subordination identity:

$$
e^{-\sigma \sqrt{\Delta_{0}}}=\frac{\sigma}{2 \sqrt{\pi}} \int_{0}^{\infty} e^{-\frac{\sigma^{2}}{4 t}} e^{-t \Delta_{0}} \frac{\mathrm{d} t}{t^{3 / 2}}
$$

so that

$$
\Delta_{0} e^{-\sigma \sqrt{\Delta_{0}}}=-\frac{\sigma}{2 \sqrt{\pi}} \int_{0}^{\infty} e^{-\frac{\sigma^{2}}{4 t}}\left(\frac{\partial}{\partial t} e^{-t \Delta_{0}}\right) \frac{\mathrm{d} t}{t^{3 / 2}} .
$$

According to [12, Corollary 5] (see also [21, Theorem 5.2.15]), the Sobolev inequality of dimension $d$ on $M_{0}$ implies

$$
\left|\frac{\partial p_{t}^{0}(x, y)}{\partial t}\right| \leq \frac{C}{t^{\frac{d}{2}+1}} e^{-c \frac{d^{2}(x, y)}{t}}, \quad \forall(x, y) \in M_{0} \times M_{0}, \forall t>0,
$$

where $p_{t}^{0}(x, y)$ is the heat kernel on $M_{0}$. So, if $\Omega$ is an open set and $F$ a compact set such that $d(F, \Omega) \geq \varepsilon>0$, then

$$
\left|\frac{\partial p_{t}^{0}(x, y)}{\partial t}\right| \leq \frac{C}{t^{\frac{d}{2}+1}} \exp \left(-c \frac{\varepsilon^{2}}{t}\right), \quad \forall t>0, \forall x \in F, \forall y \in \Omega
$$


We claim that the estimates (3.7) and (3.8) imply the existence of a constant (depending on the lower bound on the Ricci curvature of $M$ and of $\delta$ ) such that, if $t \leq 1$,

$$
\left\|\frac{\partial}{\partial t} e^{-t \Delta_{0}}\right\|_{L^{p}\left(M_{0} \backslash A_{\delta}\right) \rightarrow L^{\infty}(A)} \leq C .
$$

Indeed, denoting $k_{t}(x, y)=\frac{1}{t^{\frac{d}{2}+1}} \exp \left(-c \frac{d^{2}(x, y)}{t}\right)$, and $K_{t}$ the operator with kernel $k_{t}$, then

$$
K_{t}: L^{1}(\Omega) \rightarrow L^{\infty}(F)
$$

is uniformly bounded when $t \rightarrow 0$ : this comes from the fact that for $t \leq 1$,

$$
\begin{aligned}
\left\|K_{t}\right\|_{L^{1}(\Omega) \rightarrow L^{\infty}(F)} & =\sup _{x \in F, y \in \Omega} k_{t}(x, y) \\
& \leq \frac{1}{t^{\frac{d}{2}+1}} \exp \left(-c \frac{\varepsilon^{2}}{t}\right) \\
& \leq C .
\end{aligned}
$$

Furthermore,

$$
K_{t}: L^{\infty}(\Omega) \rightarrow L^{\infty}(F)
$$

is uniformly bounded when $t \rightarrow 0$. This is equivalent to the following estimate, for all $t$ small enough:

$$
\sup _{x \in F} \int_{\Omega} k_{t}(x, y) \leq C .
$$

But for $t \leq 1$ and $x \in F, y \in \Omega$, there holds

$$
k_{t}(x, y) \leq C_{1} \exp \left(-\frac{c}{2} \frac{d^{2}(x, y)}{t}\right) .
$$

We then use the fact that the volume of balls of radius $r$ is bounded by $e^{a r}$ for a certain constant $a$, since the Ricci curvature is bounded from below on $M$; therefore, we deduce that if $t$ is small enough so that for every $x \in F, y \in \Omega$,

$$
\frac{c}{2} \frac{\varepsilon}{t}>a,
$$

then by (3.12),

$$
\sup _{x \in F} \int_{\Omega} k_{t}(x, y) \leq C_{2}
$$

Finally, (3.9) is obtained by interpolation from (3.10) and (3.11). Using in addition the analyticity of $e^{-t \Delta_{0}}$ and the fact that $e^{-\frac{1}{2} \Delta_{0}}: L^{p} \rightarrow L^{\infty}$, we obtain that

$$
\left\|\frac{\partial}{\partial t} e^{-t \Delta_{0}}\right\|_{L^{p}\left(M_{0} \backslash A_{\delta}\right) \rightarrow L^{\infty}(A)}=\left\|\Delta_{0} e^{-t \Delta_{0}}\right\|_{L^{p}\left(M_{0} \backslash A_{\delta}\right) \rightarrow L^{\infty}(A)} \leq \frac{C}{1+t}, \quad \forall t>0 .
$$


In particular,

$$
\left\|\frac{\partial}{\partial t} e^{-t \Delta_{0}}\right\|_{L^{p}\left(M_{0} \backslash A_{\delta}\right) \rightarrow L^{\infty}(A)} \leq C, \quad \forall t>0 .
$$

Using (3.6), we then obtain

$$
\left\|\Delta_{0} e^{-\sigma \sqrt{\Delta_{0}}}\right\|_{L^{p}\left(M_{0} \backslash A_{\delta}\right) \rightarrow L^{\infty}(A)} \leq C, \quad \forall \sigma>0,
$$

and reminding of (3.5), we have

$$
\begin{aligned}
&\left\|\Delta_{0} e^{-\sigma \sqrt{\Delta_{0}}}\right\|_{L^{p}\left(M_{0} \backslash A_{\delta}\right) \rightarrow L^{p}(A)}+\left\|\Delta_{0} e^{-\sigma \sqrt{\Delta_{0}}}\right\|_{L^{p}\left(M_{0} \backslash A_{\delta}\right) \rightarrow L^{1}(A)} \leq \frac{C}{(1+\sigma)^{2}}, \\
& \forall \sigma>0 .
\end{aligned}
$$

Using the fact that the support of $\bar{f}_{0}(\sigma, \cdot)$ is compact and independent of $u$, we get

$$
\left\|\bar{f}_{0}(\sigma, \cdot)\right\|_{1}+\left\|\bar{f}_{0}(\sigma, \cdot)\right\|_{p} \leq \frac{C}{(1+\sigma)^{2}}\|u\|_{p}, \quad \forall \sigma>0 .
$$

\subsection{Proof of the corollaries to Theorem 1.8}

In this final subsection, we give the proofs of Corollaries 1.10, 1.11, 1.12 and 1.13.

Proof of Corollary 1.10. Using the result of H.Q. Li [18] and noticing that the conic manifold $M_{0}$ satisfies $d_{S}=\operatorname{dim}\left(M_{0}\right)>2$ and that $p_{0}>d_{S}$, we can apply Theorem 1.8 to get that the Riesz transform on $M_{1}$ is bounded on $2 \leq p<p_{0}$. The boundedness on $L^{p}$ of the Riesz transform on $M_{1}$ for $1<p<2$ follows from CoulhonDuong's result [7] and the fact that $M_{1}$ satisfies a Sobolev inequality. Now, if the Riesz transform on $M_{1}$ were bounded on $L^{p}$ for $p>p_{0}$, then applying Theorem 1.8 reversing the roles of $M_{0}$ and $M_{1}$, we would get that the Riesz transform on $M_{0}$ is bounded on $L^{q}$ for every $q \in(1, p)$, which is false by H.Q. Li's result. Therefore, the Riesz transform on $M_{1}$ cannot be bounded on $L^{p}$ for any $p>p_{0}$.

Proof of Corollary 1.11. According to [19], a complete manifold with non-negative Ricci curvature satisfies the parabolic Harnack inequality; the parabolic Harnack inequality being stable under rough isometries (see for example [16, Remark 5.5 ] and [9, Theorem 7.1]), $M$ also satisfies it. This implies in particular (see [16, Theorem 2.7]) that $M$ satisfies the volume doubling property, as well as the Gaussian upper estimate of the heat kernel $p_{t}(x, y)$ : there are two positive constants $C$ and $c$ such that for every $t>0$,

$$
p_{t}(x, y) \leq \frac{C}{V(x, \sqrt{t})} \exp \left(-c \frac{d^{2}(x, y)}{t}\right) .
$$


Given our hypothesis on the volume of geodesic balls, we get for every compact $F$, there is a constant $C(F)$ such that

$$
p_{t}(x, y) \leq \frac{C(F)}{t^{\frac{\nu}{2}}} \exp \left(-c \frac{d^{2}(x, y)}{t}\right), \quad \forall x \in F, \forall y \in M, \forall t \geq 1 .
$$

Also, for small time, if $n$ denotes the dimension of $M$, we have

$$
p_{t}(x, y) \leq \frac{C(F)}{t^{\frac{n}{2}}} \exp \left(-c \frac{d^{2}(x, y)}{t}\right), \quad \forall x \in F, \forall y \in M, \forall t \leq 1 .
$$

In [12, Theorem 3] implies that for every $x \in F, y \in M$ and $t \geq 1$,

$$
\begin{aligned}
\left|\frac{\partial p_{t}(x, y)}{\partial t}\right| & \leq \frac{C(F)}{t^{\frac{v}{4}+1} V(y, \sqrt{t})^{\frac{1}{2}}} \exp \left(-c \frac{d^{2}(x, y)}{t}\right) \\
& \leq \frac{C(F)}{t^{\frac{v}{4}+1}(\sqrt{t}+d(x, y))^{\frac{v}{2}}} \exp \left(-c \frac{d^{2}(x, y)}{t}\right) \\
& \leq \frac{C(F)}{t^{\frac{v}{2}+1}} \exp \left(-\frac{c}{2} \frac{d^{2}(x, y)}{t}\right) .
\end{aligned}
$$

For small time, we get by the same argument that

$$
\left|\frac{\partial p_{t}(x, y)}{\partial t}\right| \leq \frac{C(F)}{t^{\frac{n}{2}+1}} \exp \left(-\frac{c}{2} \frac{d^{2}(x, y)}{t}\right), \quad \forall x \in F, \forall y \in M, \forall t \leq 1 .
$$

Thus, as in the proof of Theorem 1.8 for one end, we get estimate (3.9), i.e. for every $t>0$,

$$
\left\|\frac{\partial}{\partial t} e^{-t \Delta_{0}}\right\|_{L^{p}\left(M_{0} \backslash A_{\delta}\right) \rightarrow L^{\infty}(A)} \leq C .
$$

Also, the hypothesis on the volume of balls implies (see [4]) that for every compact set $F$ in $M$, for all $1 \leq p \leq q \leq \infty$, and for every $t \geq 1$,

$$
\left\|e^{-t \Delta}\right\|_{L^{p}(F) \rightarrow L^{q}(M)} \leq \frac{C_{K}}{t^{v\left(\frac{1}{p}-\frac{1}{q}\right)}} .
$$

Finally, we see that the proof of Theorem 1.8 applies, which, together with Bakry's result asserting that the Riesz transform on a manifold with non-negative Ricci curvature is bounded on $L^{p}$ for every $1<p<\infty$, gives that the Riesz transform on $M$ is bounded on $L^{p}$ for every $2 \leq p<\infty$. As previously explained, $M$ satisfies the doubling property, as well as a Gaussian upper-bound for the heat kernel, and therefore, according to [7] , the Riesz transform on $M$ is bounded on $L^{p}$ for every $1<p<2$. 
Proof of Corollary 1.12. It is known by [1] that the Riesz transform on a simplyconnected nilpotent Lie group is bounded on $L^{p}$ for every $1<p<\infty$. Also, a simply-connected nilpotent Lie group has only one end. The Sobolev inequality on a simply connected, nilpotent Lie group is proved in [10, page 56]. The boundedness on $L^{p}$ of the Riesz transform on $M$ for $1<p<2$ follows from Coulhon-Duong's result [7] and the fact that $M$ satisfies a Sobolev inequality. Finally, we can apply Theorem 1.8 to get that the Riesz transform on $M$ is bounded on $L^{p}$ for $2 \leq p<\infty$.

Proof of Corollary 1.13. By an interpolation argument, it is enough to prove that the Riesz transform on $M$ is not bounded on $L^{p}$ for $n<p<q$. We proceed by contradiction: let us assume that the Riesz transform on $M$ is bounded on $L^{p}$ for a certain $n<p<q$. Then, since $M$ is $q$-hyperbolic according to Corollary 2.7, by applying Theorem 1.8 we find that the Riesz transform on $M \# M$ is bounded on $L^{r}$, for some $n<r<p$. But $M \# M=\left(\mathbb{R}^{n} \# \mathbb{R}^{n}\right) \#(N \# N)$, and since $M \# M$ is also $q$-hyperbolic, Theorem 1.8 implies that the Riesz transform on the disjoint union of $\mathbb{R}^{n} \# \mathbb{R}^{n}$ and of $N \# N$ is bounded on $L^{s}$, for some $n<s<r$. But we know, according to [5] that the Riesz transform on $\mathbb{R}^{n} \# \mathbb{R}^{n}$ is not bounded on $L^{s}$ if $s \geq n$; hence a contradiction.

\section{References}

[1] G. AleXopoulos, An application of homogeneisation theory to harmonic analysis: Harnack inequalities and Riesz transforms on Lie groups of polynomial growth, Canad. J. Math. 44 (1992) 691-727.

[2] D. BAKRY, Étude des transformations de Riesz dans les variétés Riemanniennes à courbure de Ricci minorée, In: "Lecture Notes in Mathematics", 1123, Séminaire de Probabilités XIX 351 (1983-1984), 45-174.

[3] G. Carron, Une suite exacte en $L^{2}$-cohomologie, Duke Math. J. 95 (1998), 343-371.

[4] G. CARron, Riesz transforms on connected sums, Ann. Inst. Fourier (Grenoble) 57 (2007), 2329-2343.

[5] G. Carron, T. Coulhon and A. Hassell, Riesz transform and $L^{p}$-cohomology for manifolds with Euclidean ends, Duke Math. J. 133 (2006), 59-93.

[6] T. Coulhon, and N. Dungey, Riesz transform and perturbation, J. Geometric Anal. 17 (2007), 213-226.

[7] T. CoulHon and X. T. Duong, Riesz transforms for $1 \leq p \leq 2$, Trans. Amer. Math. Soc. 351 (1999), 1151-1169.

[8] T. COUlHon, I. Holopainen and L. S AlOFF-Coste, Harnack inequality and hyperbolicity for the p-Laplacian with applications to quasi-regular mappings, Geom. Funct. Anal. 11 (2001), 1139-1191.

[9] T. COUlHON and L. SAlOFF-Coste, Variétés riemanniennes isométriques à l'infini, Rev. Mat. Iberoam. 11 (1995), 687-726.

[10] T. Coulhon, L. Saloff-Coste and N. Varopoulos, "Analysis and Geometry on Groups", Cambridge University Press, 1993.

[11] T. COULHON and Q. ZHANG, Large time behavior of heat kernel on forms, J. Differential Geom. 77 (2007), 353-384. 
[12] E. B. DAVIES, Non-Gaussian aspects of heat kernel behaviour, J. London Math. Soc. 55 (1997), 105-125.

[13] B. DEVYVER, A Gaussian estimate for the heat kernel on differential forms and application to the Riesz transform, Math. Ann. 358 (2014), 25-68.

[14] V. GoL'DSHTEIN and M. TROYANOV, Capacities in metric spaces, Integral Equations Operator Theory 44 (2002), 212-242.

[15] A. GRIGOR'YAN, "Heat Kernel and Analysis on Manifolds”, AMS/IP, 2009.

[16] A. GRIGOR' YAN, L. S ALOFF-COSTE, Stability results for Harnack inequalities, Ann. Inst. Fourier (Grenoble) 55 (2005), 825-890.

[17] C. Guillarmou and A. HASSEll, The resolvent at low energy and Riesz transform for Schrödinger operators on asymptotically conic manifolds, Part I, Math. Ann. 341 (2008), 859-896.

[18] H. Q. LI, Estimations du noyau de la chaleur sur les variétés coniques et applications, Bull. Sci. Math. 124 (2000), 365-384.

[19] P. LI and S. T. YAU, On the parabolic kernel of the Schrödinger operator, Acta Math. 156 (1986), 153-201.

[20] S. Pigola, M. Rigoli and A. Setti, "Vanishing and Finiteness Results in Geometric Analysis", Birkhäuser, 2008.

[21] L. S Aloff-Coste, "Aspects of Sobolev-Type Inequalities", Cambridge University Press, 2002.

[22] E. M. STEIN, "Topics in Harmonic Analysis Related to the Littlewood-Paley Theory", Annals of Mathematics Studies, Vol. 63, Princeton University Press, Princeton, NJ, 1970.

[23] M. TAYLOR, "Partial Differential Equations , Vol. I-III", Applied Math. Sciences, Vol. 115117, Springer-Verlag, New York, 2nd ed., 2011.

[24] M. Troyanov, Solving the p-Laplacian on manifolds, Proc. Amer. Math. Soc. 128 (2000), $541-545$

[25] N. VAROPOUlos, Hardy-Littlewood theory for semigroups, J. Funct. Anal. 63 (1985), 240260.

Laboratoire de Mathématiques Jean Leray

Université de Nantes, France

baptiste.devyver@univ-nantes.fr 